\title{
MAGNETORHEOLOGICAL FLUID SEAL WITH MINIMUM FRICTION TORQUE
}

\author{
M. Kubík , O. Macháček, I. Mazůrek
}

\begin{abstract}
The paper deals with design and tests of MagnetoRheological Fluid seal (MRFs) using innovative conception of magnetic circuit. The common design of magnetic seal uses ferrofluid. Low friction torque and low burst pressure are typical for this kind of seals. Replacement of the ferrofluid by magnetorheological fluid increases the burst pressure; however, the friction torque increase too. The optimum for sealing is low friction torque and high burst pressure. The new design of MRFs described in this paper meets both requirements; is based on gradient-pinch mode. The friction torque and burst pressure of this type of design and common design of MRFs were tested and compared. The new design of MRFs achieved 20 times lower friction torque that current solution MRFs. The developed MRFs combines the benefits of common FFs and MRFs.
\end{abstract}

\section{Keywords: magnetorheological fluid seal, MR fluid seal, magnetic seal, magnetic shaft seal,} liquid o-ring

\section{Introduction}

Seals with magnetic fluid create a liquid barrier between sealed zones; therefore, it is often called a liquid o-ring. The magnetic field keeps magnetic fluid in sealed gap. Typically, it is used for sealing of rotational shaft (Matuszewski and Szydło, 2008). The common design of seal with magnetic fluid contains of two ferromagnetic pole pieces, permanent magnet, magnetic fluid, and magnetically permeable shaft, see Fig. 1. The magnetic fluid is located between pole piece and ferromagnetic shaft, see Fig. 1.
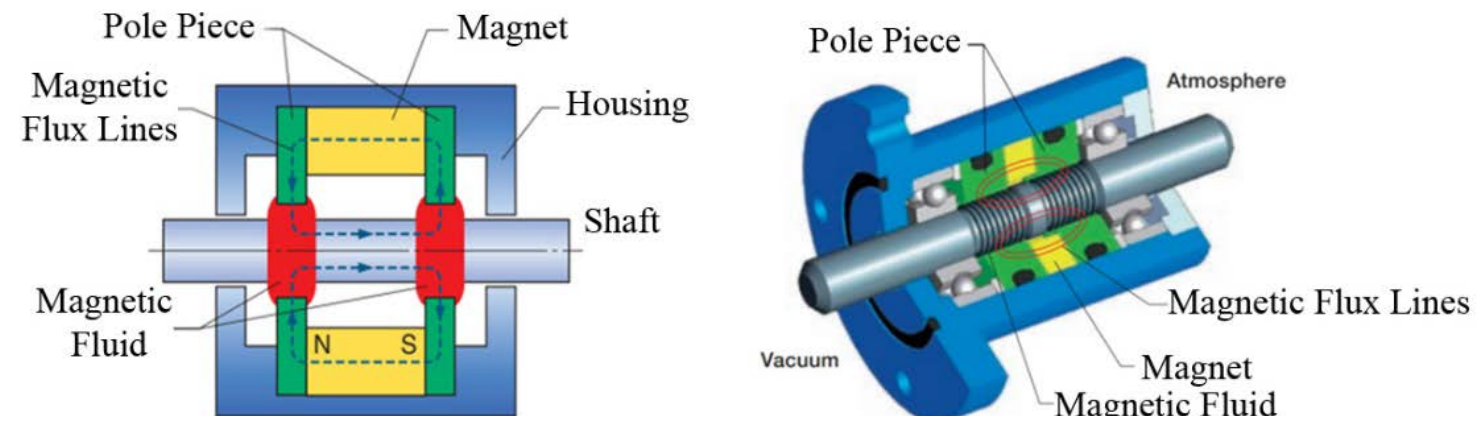

Fig. 1: Common design of magnetic fluid seal (EagleBurgmann, n.d., modified).

The magnetic fluid is composed of ferromagnetic particles, carrier fluid and additives. These fluids can be divided into the two groups by the size of particles which are used: Magnetorheological fluid contains microparticles and Ferrofluids are made of nanoparticles. The magnetic seals are divided by the fluid which is used into: Ferro Fluid seals (FFs) and MagnetoRheological Fluid seals (MRFs)(Kordonski and Gorodkin, 1996).

\footnotetext{
*All autors: Institute of Machine and Industrial Design, Brno University of Technology, Technická 2896/2;616 69, Brno; CZ, michal.kubik@vutbr.cz
} 


\section{FerroFluid seals (FFs)}

Ferro Fluid seal uses magnetic fluid which is composed of ferromagnetic nanoparticles. Ferrofluid seal is characterized by small friction torque (Fig. 2 right, number 3) and high level of tightness. Considering above advantages, ferrofluid seal is appropriate for sealing ultrahigh vacuum, highly clean and corrosive environments (Matuszewski and Szydło, 2008). However, the disadvantage is relative low burst pressure. Burst pressure is a maximum pressure drop over which the leakage is observed. Typical burst pressure of FFs is in tens of $\mathrm{kPa}$, see Fig. 2 left, number 2. This sealing system used a wide range of equipment worldwide, e.g. vacuum-rotary feedthrough, fluid seals for fans and blowers or hard disk drive spindle.
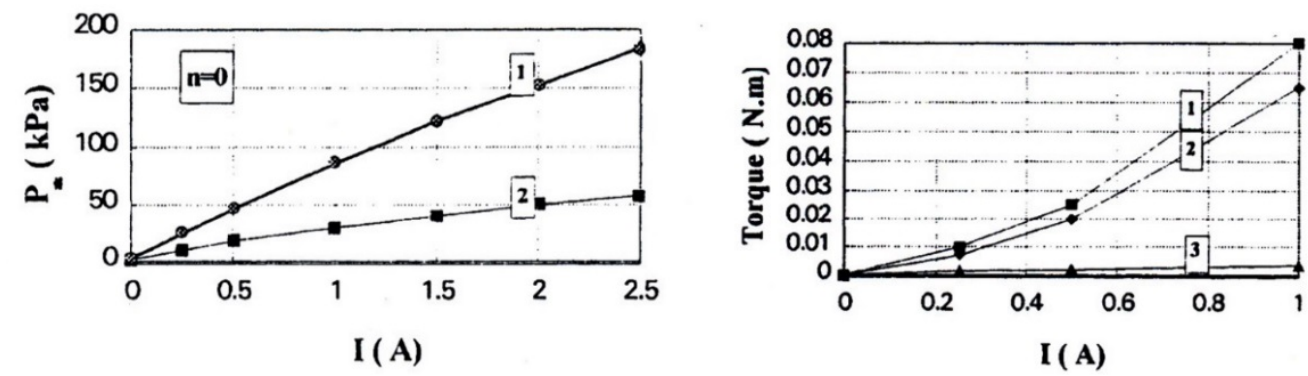

Fig. 2: Static burst pressure of MRFs (1) and FFs (2), left; friction torque in MRFs (1) 81 rpm and (2) 27 rpm, and friction torque in FFs (3) 81 rpm, right (Kordonski and Gorodkin, 1996).

\section{MagnetoRheological Fluid seals (MRFs)}

MagnetoRheological Fluid seal uses magnetorheological (MR) fluid which is composed of ferromagnetic microparticles, usually in range from 1 to $10 \mu \mathrm{m}$. The main motivation of using bigger particles is higher magnetization (Matuszewski and Szydło, 2008). This causes significant increase of burst pressure of seal. Typical burst pressure of MRFs is in hundreds of $\mathrm{kPa}$, see Fig. 2 left, number 1 . The second motivation to use MRFs is in MR devices, as magnetorheological damper (Kubík et al., 2017; Strecker et al., 2015) or clutch (Imaduddin et al., 2015),because the same fluid is used for damping and sealing at the same time. The disadvantage of MRFs is high friction torque compared to FFs, see Fig. 2 right, number 1. This is caused by tearing the chains of microparticles.

\section{Aim of research}

The main aim of our research was to develop a design of magnetic fluid seal which provide greater burst pressure than FFs and lower friction torque in than common MRFs.

\section{Materials and Methods}

\subsection{New type of magnetorheological fluid seal}

Magnetic circuit of new type of MRFs was designed by magnetostatic model, the circuit arrangement is based on gradient-pinch mode of MR fluid which published (Goncalves and Carlson, 2009) or (Gołdasz and Sapiński, 2017). Design of gradient-pinch MRFs is composed of two pole pieces, electromagnetic coil, MR fluid and nonmagnetic shaft with diameter $18 \mathrm{~mm}$, see Fig. 3. This design is specific in that the ferromagnetic particles creates chain link between two pole pieces when electric current is applying on the coil, see Fig. 3. There is no tearing of particles chain and the lower friction torque is expected.

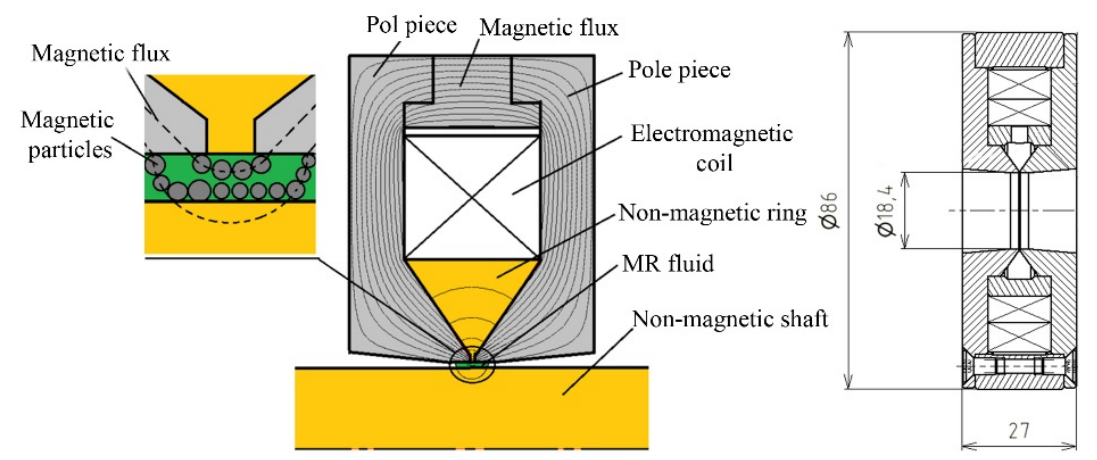

Fig. 3: Design of gradient-pinch MRFs; function principle (left); manufactured design (right). 
The sealing effect is ensured by pressing the chains of particles on surface of the shaft. In the next sections, this design is called gradient-pinch MRFs. For the comparison of properties, the common design of MRFs was also tested, see Fig. 1.

\subsection{Experimental setup}

New test device was developed for testing friction torque and burst pressure of both above mentioned magnetic seal, see Fig. 4. The test device is composed of shaft, which is supported by two bearings, shaft rubber seal, tubus and magnetic sealing. Lord MRF-132DG was used for experiments.

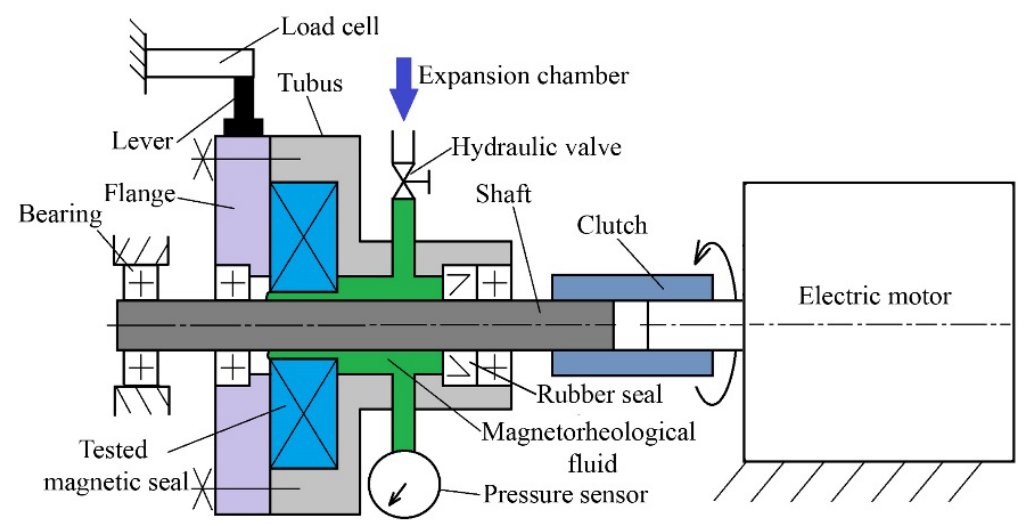

Fig. 4: Developed test device.

\section{Friction torque measurement method}

The test device was set as rotatable. Test device was driven by an electric motor via the clutch, see Fig. 4 . During rotation of the shaft, the magnetic seal and whole test device create friction torque. The value of friction torque was determined from measured force at load cell HBM DF2SR-3 and known length of lever. The measured friction torque is composed of friction torque of MRFs and friction torque of test devices. For this reason, the friction torque of MRFs was determined as difference between measured friction torque and friction torque of test device. The measurements was provided at frequency of rotation $20 \mathrm{~Hz}\left(1200 \mathrm{~min}^{-1}\right)$ for different electric current in range $0.3 \mathrm{~A}$ to $2 \mathrm{~A}$. The presented friction torque was evaluated as mean value of 15 second of experiment. The pressure in the fluid was atmospheric during the measurement.

\section{Burst pressure measurement method}

The expansion chamber with hydraulic valve and pressure sensor P8AP/20 bar were connected to test device. Hydraulic system with MRFs seal was pressurized by expansion chamber. The pressure in the fluid was gradually increased during experiment. The burst pressure was measured as maximum pressure before a leak of the MR fluid was occurred, because even a little leakage causes rapid decrease of pressure in hydraulic system. The burst pressure of seal was measured in static regime $(0 \mathrm{~Hz})$ for different current applied on the coil in the range from 0 A to $1 \mathrm{~A}$. However, the influence of rotation of shaft on burst pressure was measured with constant current $2 \mathrm{~A}$.

\section{Results}

The friction torque of gradient-pinch MRFs (Fig. 5 left, circle) and common MRFs (Fig. 5 left, square) were compared for several current in the coil. The friction torque of gradient-pinch MRFs is independent on electric current and average value was $12 \mathrm{~N} . \mathrm{mm}$. On the other hand, the friction torque of common MRFs is strongly dependent on electric current. Gradient-pinch MRFs achieved up to 25 times lower torque moment than common design. The static burst pressure dependency on electric current of the both MRFs were measured and compared in Fig. 5 right. The common design of MRFs achieved approximately 2 times higher burst pressure than new design. The increase of burst pressure of gradientpinch MRFs between $0 \mathrm{~A}$ to $0.4 \mathrm{~A}$ is low. Probably because of the ferromagnetic particles are not sufficiently pressed on the shaft surface at low electric current. After exceeding $0.4 \mathrm{~A}$, a significant increase of burst pressure was observed. 

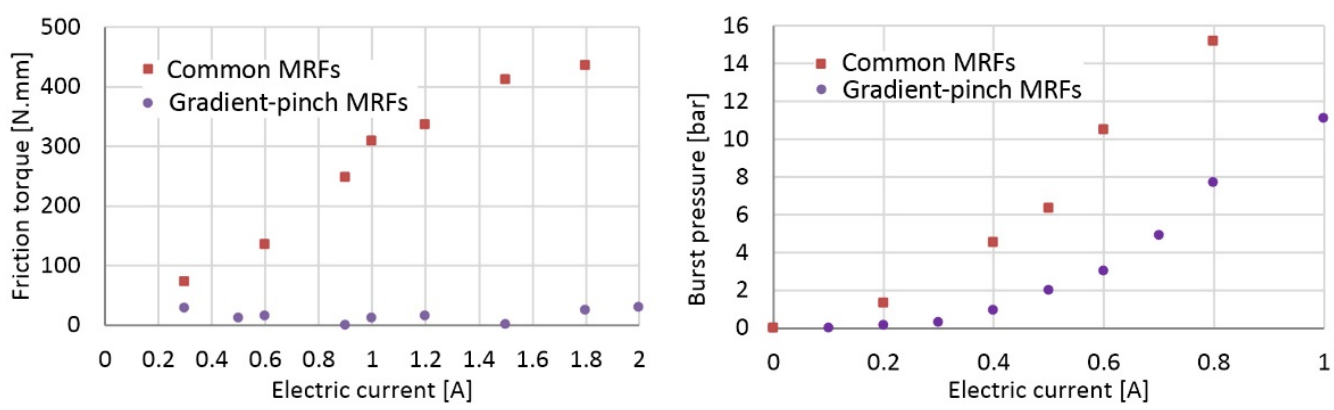

Fig. 5: Measured friction torque of new MRFs (circle) and common MRFs (square), left; measured static burst pressure, right.

The influence of shaft rotation on burst pressure gradient-pinch MRFs at electric current 2 A was measured, see Fig. 6. The burst pressure significantly decreases with increasing shaft rotation. A similar trend was also observed in common MRFs and FFs, according to (Kordonski and Gorodkin, 1996).

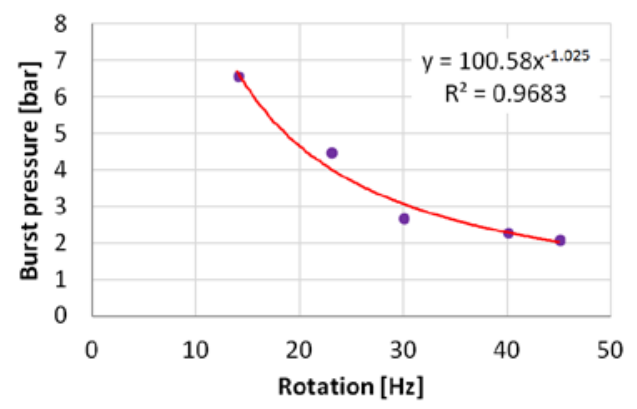

Fig. 6: Influence of rotation to burst pressure of gradient-pinch MRFs.

\section{Conclusion}

Gradient-pinch MRFs achieved significantly lower friction torque (25 times), but the burst pressure is also lower (2 times) than common version of MRFs. However, the static burst pressure of new design is significantly higher than 1 bar which is usual for FFs. The burst pressure is strongly influenced on rotation of shaft in gradient-pinch MRFs, according to experiments. The proposed design combines advantages between FFs and common MRFs.

\section{Acknowledgement}

This experiment could be provided thanks to the kind sponsorship of various grants and numerous agencies. We would like to thank explicitly to the GAČR 17-10660J, GAČR 17-26162S and FSI-S-174428.

\section{References}

EagleBurgmann (n.d.) Magnetic fluid seal. Available from: www.ekkeagle.com.

Gołdasz, J. and Sapiński, B. (2017) Magnetostatic analysis of a pinch mode magnetorheological valve. 11(3), 229232.

Goncalves, F.D. and Carlson, J.D. (2009) An alternate operation mode for MR fluids - magnetic gradient pinch. Journal of Physics: Conference Series 149.

Imaduddin, F., Nizam, M. and Mazlan, S.A. (2015) Response of a magnetorheological brake under inertial loads. $7(2), 308-322$.

Kordonski, W.I. and Gorodkin, S.R. (1996) Magnetorheological fluid-based seal. Journal of Intelligent Material Systems and Structures, 7(5), 569-572.

Kubík, M., Macháček, O., Roupec, J., Strecker, Z. et al. (2017) Design and testing of magnetorheological valve with fast force response time and great dynamic force range. Smart Materials and Structures, 26(4), 1-10.

Matuszewski, L. and Szydło, Z. (2008) The application of magnetic fluids in sealing nodes designed for operation in difficult conditions and in machines used in sea environment. 15(3), 49-58.

Strecker, Z., Roupec, J., Mazůrek, I., et al. (2015) Design of magnetorheological damper with short time response. Journal of Intelligent Material Systems and Structures, 26(14), 1951-1958. 